\title{
Automatic Evaluation of Test Strips for Anti-Ganglioside Antibodies in Patients with Guillain-Barré Syndrome Using EUROLineScan Software
}

\author{
Chetana Vaishnavi*, Chitralekha Behura, Sudesh Prabhakar \\ Departments of Gastroenterology and Neurology, Postgraduate Institute of Medical Education and Research, \\ Chandigarh, India \\ Email: ${ }^{*}$ cvaishnavi@rediffmail.com, ${ }^{*}$ chetanavaishnavi@gmail.com
}

Received 28 July 2014; revised 20 August 2014; accepted 12 September 2014

Copyright (C) 2014 by authors and Scientific Research Publishing Inc.

This work is licensed under the Creative Commons Attribution International License (CC BY). http://creativecommons.org/licenses/by/4.0/

cC) (i) Open Access

\begin{abstract}
Campylobacter jejuni infection has been implicated in the pathogenesis of Guillain-Barré syndrome (GBS) due to production of humoral immune response against neural antigens. A case-control study was performed in a tertiary care teaching hospital for the estimation of anti-ganglioside antibodies in GBS patients and their controls. Blood samples were collected from 59 GBS cases, 58 neurological controls (NC) and 60 non-neurological control (NNC) patients for automatic estimation of IgG and IgM antibodies to seven gangliosides using EUROLineScan software. Antibodies of IgG class for GM1 were highly significant in GBS $(p=0.000)$ and $N C(p=0.031)$ compared to NNC. However GBS group was not significant $(p=0.413)$ compared to NC. For GM2 ganglioside, GBS and NC groups were significant $(p=0.000)$ compared to NNC, but GBS group was not significant $(p=$ 0.999) compared to NC. For GM3 ganglioside, GBS and NC groups were significant $(p=0.000)$ compared to NNC; but GBS group was insignificant $(p=0.858)$ compared to NC with similar trend for all other ganglioside antibodies. When IgM class of antibodies was evaluated for GM1, GBS group was not significant $(p=0.604)$ whereas $N C$ group was significant $(p=0.000)$ compared to NNC. GBS group was not significant compared to NC $(p=0.011)$. The trend was the same for GM2 antibodies. For GM3, GBS group was significant $(p=0.010)$ and NC was near significant $(p=0.055)$ compared to NNC. However GBS group was not significant $(p=0.808)$ compared to NC. No groups were significant $(p>0.05)$ in relation to the remaining gangliosides except for GQ1b where GBS group $(p=0.001)$ and NC group were significant $(p=0.000)$ compared to NNC. GBS group was also significant $(p=0.001)$ compared to $N C$ and NNC. Anti-gangliosides antibodies were present in highly significant levels in the GBS group, though they were also present in the non-paralytic neurological control patients compared to the non-neurological control group.
\end{abstract}

\footnotetext{
"Corresponding author.
}

How to cite this paper: Vaishnavi, C., Behura, C. and Prabhakar, S. (2014) Automatic Evaluation of Test Strips for AntiGanglioside Antibodies in Patients with Guillain-Barré Syndrome Using EUROLineScan Software. Advances in Microbiology, 4, 890-898. http://dx.doi.org/10.4236/aim.2014.413099 


\section{Keywords}

\section{Anti-Ganglioside Antibodies, C. Jejuni, Guillain-Barré Syndrome}

\section{Introduction}

Guillain-Barré syndrome (GBS) is an acute, autoimmune serious disorder of the peripheral nerves characterized by ascending paralysis. This syndrome is usually triggered by an acute infectious agent in $50 \%-70 \%$ of cases after an interval of 1 to 6 weeks [1]. The implicated organisms include Campylobacter jejuni, Mycoplasma pneumoniae, hepatitis B virus, cytomegalovirus, varicella zoster virus, Epstein Barr virus, rubeola and human immunodeficiency virus. The targets of such immune attack are thought to be gangliosides present in large quantities on human nerve tissues. GBS has been reported to occur sporadically also in association with a number of vaccines such as oral polio vaccine [2] rubella vaccination in combined vaccine [3] and tetanus toxoid [4], but the relation may be casual rather than causal as population studied do not support a well documented role in causing GBS. It is possible that wide scale immunization with any foreign antigen might evoke the same increased incidence of disease in those individuals predisposed to this complication.

Although its cause and pathogenesis remain ill defined, recent studies have suggested that $C$. jejuni may be the most common preceding pathogen [1] [5]. In fact GBS has been observed as an important sequelum of $C$. jejuni infections. Rhodes and Tattersfield [6] were the first to report a patient who developed GBS 19 days after C. jejuni infection. Other reports soon followed confirming the association of GBS preceded by C. jejuni infection. Prior to 1982 routine culture of stools for this species was not done explaining the reason for $C$. jejuni not being associated with GBS until then [7]. In a retrospective serological study, Kaldor and Speed [8] found that $38 \%$ of patients with GBS had recent $C$. jejuni infection which results from demyelination of peripheral nerves. GBS is generally precipitated 1 - 3 weeks following $C$. jejuni infection which suggests a humoral immunopathogenic mechanism.

Autoantibodies against gangliosides are found in patients with peripheral neuropathies, including GBS, chronic inflammatory demyelinating polyneuropathy, multifocal motor neuropathy (MMN) and in Miller-Fisher syndrome (MFS), a subtype of GBS. The clinical significance of ganglioside antibodies have been reported in literature. In GBS both IgM and IgG against GM1, GD1a and GT1b and only IgG antibodies against GQ1b are found in MFS [9]. In case of MMN, IgM antibodies against GM1 ganglioside are found [10] and with sensory neuropathy IgG antibodies against GD1b are found [11]. In a previous prospective examination of GBS patients, the stool culture yielded Campylobacter of Penner group O:19 in 5.7\% of them with high intensity of anti-ganglioside antibodies [12]. However the intensity of anti-ganglioside antibodies was read visually by color reaction and was therefore subjective. Software made available by EUROLineScan was used in the present investigation for automatic estimation of test strips for ganglioside antibodies in GBS patients and their controls. The EUROLineScan Software is used to identify and quantitate the bands for human antibodies of class IgG and IgM to the seven gangliosides GM1, GM2, GM3, GD1a, GD1b, GT1b and GQ1b in serum samples. The incubated test strips are scanned using a flat-bed scanner with a protocol sheet or a template. EUROLineScan recognizes the position of the test strips, identifies the bands and measures their intensity. A separate result sheet can be produced for each patient.

\section{Materials and Methods}

A total of 177 patients including cases and controls attending the Neurology outpatient department or emergency and other wards at the Post Graduate Institute of Medical Education and Research, Chandigarh, India were enrolled for the prospective investigation. All patients underwent a detailed enquiry and clinical examination. Clinical features of each patient along with primary diagnosis were recorded in a pre-printed proforma. The project was approved by the Institute Ethical Committee and informed consent was taken from all the patients involved in the study. The study was conducted between October 2006 and November 2009 as per the study design given (the flowing diagram).

\subsection{Study Population}

The study population was divided into 3 groups as follows: 


\section{Study Design}

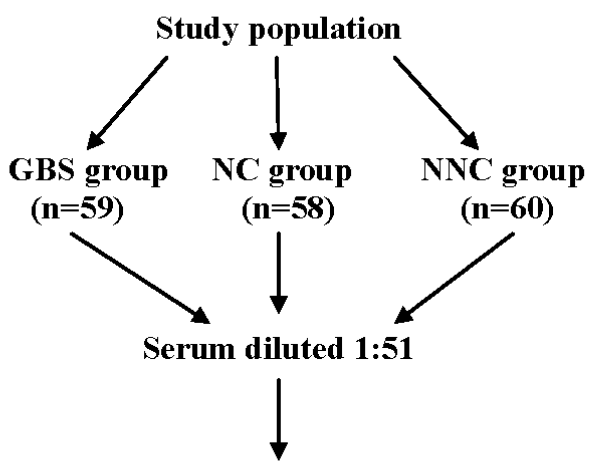

Incubated with test strips coated with parallel lines of purified antigens

(GM1, GM2, GM3, GD1a, GD1b, GT1b and GQ1b) at $25^{\circ} \mathrm{C}$ for $60 \mathrm{~min}$. on a shaker<smiles>[3H][13CH3]</smiles>

Washed $3 \times 5 \mathrm{~min}$. each with $1.5 \mathrm{ml}$ buffer on a shaker<smiles>[13CH2][13CH3]</smiles>

Incubated with $1.5 \mathrm{ml}$ Alkaline phosphatase conjugated anti-human IgG or IgM at $25^{\circ} \mathrm{C}$ for $60 \mathrm{~min}$. on a shaker<smiles>C[13CH2]</smiles>

Wash cycles repeated $(3 \times 5$ min. each $)$<smiles>[3H][13CH3]</smiles>

Incubated with substrate solution at $25^{\circ} \mathrm{C}$ for $10 \mathrm{~min}$. on a shaker<smiles>C[13CH2]</smiles>

Liquid from each channel aspirated off and washed $(3 \times 1 \mathrm{~min}$. each) with distilled water

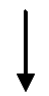

Test strips placed on evaluation protocol, dried and evaluated using EUROLineScan

(i) GBS group: This group comprised of 59 GBS patients who underwent nerve conduction studies to establish the diagnosis.

(ii) Neurological control (NC) group: This group involved 58 patients with non-paralytic neurological disease and served as controls to the GBS group.

(iii) Non-neurological control (NNC) group: In this group 60 non-neurological patients taken from various wards of the hospital and who acted as controls to both the GBS and the NC groups were included.

The inclusion criteria in GBS group were those patients who underwent nerve conduction studies to establish the diagnosis. In the NC group patients with non-paralytic neurological diseases were included. In the NNC group patients with non-neurological ailments and taking treatment from various wards of the hospital were included. Pregnant women and very young children were excluded from the investigation. 


\subsection{Clinical Diagnosis of GBS Patients}

The clinical diagnosis of the GBS patients was based on the diagnostic criteria by Asbury and Cornblath [13]. Nerve conduction studies involved the motor (median and ulnar) nerves in the upper limbs and the common peroneal and tibial nerves in the lower limb. The sensory nerves examined were superficial peroneal or sural nerves in the lower limbs. The electrophysiological studies involved distal latency, compound muscle action potential and conduction velocity of motor nerves and peak latency, sensory nerve action potential and conduction velocity of sensory nerves. $\mathrm{F}$ waves were recorded from median and tibial nerves and persistence and latencies were taken account of.

\subsection{Automatic Evaluation of Test-Strips for Anti-Ganglioside Antibodies}

Five milliliters of blood samples were drawn from each patient for anti-ganglioside antibody assay. Sera separated from the blood samples of both cases and controls were tested for the presence of IgG and IgM classes of antibodies to gangliosides using commercially available EUROLINE test system (Lubeck, Germany) as described earlier [12]. The EUROLINE is a test system for fast and reliable determination of multiple ganglioside antibodies in patient sera using immunodot method [14]. The test kit contains test strips coated with parallel lines of purified antigens. The blot strips are included in the first reaction of step with diluted patient serum. In the case of positive samples, specific antibodies of the class IgG or IgM will bind to the antigens. To detect the bound antibodies, a second incubation is carried out using an enzyme-labeled anti-human IgG or IgM which promotes a colour reaction.

Briefly, each serum diluted 1:51 in buffer was incubated with membrane test strips printed with purified ganglioside fraction (GM1, GM2, GM3, GD1a, GD1b, GT1b and GQ1b) and rocked for 1 hour for binding to their specific antigens. After washing with buffer solution, enzyme conjugate was added and again rocked for 1 hour. Wash cycles with buffer solution were repeated 3 times and substrate solution was added to it. After rocking for 10 minutes the strips were again washed. Automatic estimation of test strips for anti-ganglioside antibodies were done using EUROLineScan software supplied by the manufacturer.

\subsection{Statistical Analysis}

Data were entered into the data base program SPSS version 16.0, for intra and inter groups comparison. Data was expressed as mean \pm standard deviation. The analysis of intensity was done using one way analysis of variance (ANOVA) followed by Tukey's post Hoc test. p value $<0.05$ was considered as significant.

\section{Results}

There were 37 males and 22 females with age range 9 - 94 years in the GBS group. In the NC group there were 35 males and 23 females with age range 13 - 80 years and in the NNC group there were 30 males and an equal number of females with age range 16 - 75 years. The mean and range of anti-ganglioside antibodies of both IgG and IgM classes are given in Table 1. Comparison of IgG and IgM classes of anti-ganglioside antibodies between different groups are given in Table 2. Antibodies of IgG class (Figure 1) for GM1 was highly significant in GBS $(p=0.000)$ and NC ( $=0.031)$ groups compared to NNC group. However GBS group was not significant ( $p=0.413)$ compared to NC. For GM2 ganglioside, GBS and NC groups were significant $(p=0.000)$ compared to NNC, but GBS group was not significant $(\mathrm{p}=0.999)$ compared to NC. For GM3 ganglioside, GBS and NC groups were significant $(p=0.000)$ compared to NNC; but GBS group was insignificant $(p=0.858)$ compared to NC with similar trend for all other anti-ganglioside antibodies. Only for GT1b, GBS was near significance $(\mathrm{p}=0.051)$ when compared to NC group.

When IgM class of antibodies was evaluated (Figure 2) for GM1, GBS group was not significant ( $\mathrm{p}=0.604$ ) whereas NC group was significant $(\mathrm{p}=0.000)$ compared to NNC. GBS group was not significant compared to NC $(p=0.011)$. The trend was the same for GM2 antibodies. For GM3, GBS group was significant $(p=0.010)$ and NC group was near significant $(\mathrm{p}=0.055)$ compared to NNC. However GBS group was not significant $(\mathrm{p}=$ $0.808)$ compared to NC. No group was significant $(p>0.05)$ in relation to the remaining gangliosides except for GQ1b, where GBS group $(p=0.001)$ and NC group were significant $(p=0.000)$ compared to NNC. GBS group was also significant $(\mathrm{p}=0.001)$ compared to NC and NNC groups. 
Table 1. The mean and range of each ganglioside antibodies of both IgG and IgM classes.

\begin{tabular}{ccccccccccccccccc}
\hline Groups & \multicolumn{2}{c}{ GM1 } & \multicolumn{2}{c}{ GM2 } & \multicolumn{2}{c}{ GM3 } & \multicolumn{2}{c}{ GD1a } & \multicolumn{2}{c}{ GD1b } & \multicolumn{2}{c}{ GT1b } & GQ1b \\
IgG & Mean & Range & Mean & Range & Mean & Range & Mean & Range & Mean & Range & Mean & Range & Mean & Range \\
GBS-1 & 8.27 & $0-87$ & 6.96 & $0-26$ & 7.22 & $0-36$ & 5.94 & $0-27$ & 4.16 & $0-34$ & 6 & $0-43$ & 3.77 & $0-22$ \\
NC-1 & 5.68 & $0-39$ & 7.0 & $0-28$ & 6.46 & $0-28$ & 4.08 & $0-16$ & 2.77 & $0-12$ & 3.58 & $0-25$ & 4.15 & $0-16$ \\
NNC-1 & 0.533 & $0-9$ & 0.85 & $0-3$ & 0.383 & $0-5$ & 0.08 & $0-2$ & 0.1 & $0-2$ & 1.266 & $0-6$ & 0.16 & $0-1$ \\
IgM & Mean & Range & Mean & Range & Mean & Range & Mean & Range & Mean & Range & Mean & Range & Mean & Range \\
GBS-1 & 12.69 & $0-70$ & 12.52 & $0-40$ & 6.57 & $0-28$ & 4.84 & $0-23$ & 4.86 & $0-23$ & 4.76 & $0-24$ & 4.94 & $0-37$ \\
NC-1 & 6.53 & $0-40$ & 9.5 & $0-59$ & 5.82 & $0-44$ & 5.15 & $0-34$ & 5.29 & $0-22$ & 4.63 & $0-30$ & 1.65 & $0-17$ \\
NNC-1 & 14.7 & $2-28$ & 14.6 & $8-22$ & 3.05 & $0-9$ & 5.83 & $2-10$ & 5.56 & $4-8$ & 4.08 & $0-8$ & 8.33 & $0-14$ \\
\hline
\end{tabular}

Table 2. Comparison of IgG and IgM between groups ( $\mathrm{p}$ value).

\begin{tabular}{|c|c|c|c|c|c|c|}
\hline \multirow[t]{2}{*}{ Ganglioside antigens } & \multicolumn{2}{|c|}{ GBS and NC } & \multicolumn{2}{|c|}{ GBS and NNC } & \multicolumn{2}{|c|}{ NC and NNC } \\
\hline & IgG & IgM & IgG & IgM & IgG & IgM \\
\hline GM2 & 0.999 & 0.228 & 0.000 & 0.464 & 0.000 & 0.014 \\
\hline GM3 & 0.858 & 0.808 & 0.000 & 0.010 & 0.000 & 0.055 \\
\hline GD1a & 0.121 & 0.944 & 0.000 & 0.548 & 0.000 & 0.754 \\
\hline GD1b & 0.132 & 0.836 & 0.000 & 0.615 & 0.001 & 0.929 \\
\hline GT1b & 0.051 & 0.989 & 0.000 & 0.713 & 0.062 & 0.800 \\
\hline GQ1b & 0.891 & 0.001 & 0.000 & 0.001 & 0.000 & 0.000 \\
\hline
\end{tabular}

\begin{tabular}{|l|r|r|r|}
\hline \multicolumn{2}{|l|}{ EUROIMMUN } & $\begin{array}{l}\text { Medizinische } \\
\text { Labordiagnostika }\end{array}$ \\
\hline AG
\end{tabular}

Figure 1. Automatic evaluation of test strip for IgG class of anti-ganglioside antibody.

\section{Discussion}

The beneficial effect of plasmapheresis and lack of benefit from corticosteroid prove that GBS may be antibody mediated [15]. A prospective case-control study was performed to evaluate anti-ganglioside antibodies with GBS patients as the case, and patients with other neurological disorders and those with non-neurological disorders as two sets of controls. $C$. jejuni is known to stimulate production of anti-ganglioside antibodies to all the seven gangliosides studied. A total of 59 GBS cases were compared with almost an equal number each of hospital control groups comprising of NC and NNC patients. In the present study IgG class antibodies for GM1 were at highly significant level for both GBS and NC groups compared to NNC, though GBS group was not significant when compared to NC. It has been reported that antibodies against monosialoganglioside GM1 are associated with MMN with a prevalence of $40 \%-70 \%$ [10] and are in most cases of the IgM class. Anti-GM1 


\begin{tabular}{|l|r|c|c|}
\hline \multicolumn{2}{|c|}{ EUROIMMUN } & $\begin{array}{l}\text { Medizinische } \\
\text { Labordiagnostika } \\
\text { AG }\end{array}$ \\
\hline Ap2/26-85 & Intensity & Class & Automatic evaluation of test strips using the \\
EUROLineScan software
\end{tabular}

Figure 2. Automatic evaluation of test strip for IgM class of anti-ganglioside antibody.

antibodies of the IgM class were significantly high in the GBS group in the present study indicating a MMN variant of the syndrome, where the motor nerves are affected. Elevated antibody titers against GM1 also occur in GBS in $22 \%$ - $30 \%$ of the cases and correlates with the disease activity [16]. In the acute phase of the disease the titer increases to a maximum value and decreases as the disease progresses [17]. In the present study, in some of the GBS patients antibodies to gangliosides were not detected as the patients come for treatment later on during the course of the disease. Moreover Campylobacter gastroenteritis in developing countries is acquired in childhood as self-limiting or undiagnosed infections, of which GBS can be the long-term sequel. Anti-GM1 antibodies are elevated in patients with various forms of dementia, and may be responsible for the increased antibodies to this epitope seen in the NC patients.

Similarly for GM2 and GM3 ganglioside, GBS and NC groups were significant compared to NNC, but GBS group was not significant compared to NC with comparable trend for all other ganglioside antibodies. It has been suggested that IgG anti-GD1a antibody is a predictor of the motor axonal form of GBS [18]. Dikmen and Oge [19] reported recurrent GBS with GD1a anti-ganglioside antibodies in a 38-year-old woman with an uneventful health record, except for an episode of gastroenteritis one week before admission. GD1b disialoganglioside antibodies occur in rare cases of patients with sensory neuropathy whereas GQ1b tetrasialoganglioside can be detected in more than 90\% of the patients with MFS [9]. GM1, GD1a and GT1b are known to be associated with GBS [9]. A very high association of these three gangliosides was found in our patients with GBS. IgG class against GT1b was near to significance in GBS group compared to NC group. Taheraghdam et al. [20] in a prospective study of 30 GBS patients measured baseline serum samples for antibodies against GQ1b, GT1b, GD1a, GD1b, GM1, GM2, GM3. Anti-GM1 was found in 3 patients (10\%) and anti-GM3 was found only in one patient (3.3\%). They observed that the anti-ganglioside antibodies were positive in patients who suffered from severe phase of the disease.

Shared antigens between $C$. jejuni and nerve fibers have been proposed to be responsible for neuropathy in GBS. Approximately two-third patients of GBS report some or the other form of preceding infectious illness. Though one or two of these glycolipid-mimicking structures are identified on neuropathies by M. pneumoniae or cytomegalovirus, C. jejuni evokes the maximum antibodies to glycolipids. Kuroki et al. [7] reported the frequency of PENNER 19 strains isolated from patients with GBS. In an earlier study with the same patient groups [12], C. jejuni PENNER group 19 was isolated in 5.7\% of the GBS cases with none from the control groups. The reason for lower isolation of $C$. jejuni in the study was due to the fact that the mean excretion time of the organism in stools is only 16 days and GBS patients generally report much later after the onset of neuropathy. Penner serotyping in Japan and South Africa showed that GBS associated strains were commonly of serotypes O:19 and O:41 respectively. In Japan of 12 C. jejuni isolated from GBS patients, 10 were serotype O:19, whereas 0:19 represent less than $2 \%$ of $C$.jejuni isolates from patients with uncomplicated enteritis [21]-[23]. Expression of a GM1 like LOS in class A strains and GQ1b like LOS in class B strains is responsible for development of GBS and MFS respectively [24]. The class A locus and serotype O:19 seemed to be linked to sialytransferase gene (cst II) polymorphism resulting in promotion of both GM1 like and GD like structure synthesis on LOS and consequently the risk of producing anti-ganglioside antibodies and developing GBS [23] [25].

As only case-reports of GBS are being presented, the characteristics of the infected hosts or the specific 
organisms have not been examined to a great extent. It is not known whether extraintestinal infections due to subpopulation of $C$. jejuni that are capable of invading the blood-stream or of compromising host defense can lead to production of antibodies. Antibodies to gangliosides are associated with several distinct syndromes of peripheral neuropathy and are generally polyclonal in nature, but sometimes can be restricted to a single immunoglobulin class. Unlike in most other organ specific autoimmune disease, antibodies in peripheral neuropathy appear to recognize glycolipid antigens rather than protein antigen of myelin or axon.

Kaur et al. [26] described 56 cases of GBS based on clinical characteristics, biochemical investigation and electrodiagnostic study. However, no attempt was made to look for an association of GBS with C. jejuni. Vaishnavi [27] reviewed the association of C. jejuni with GBS. Earlier in India, only electrophysiological studies were carried out in patients of GBS. Serological studies that could determine the association of $C$. jejuni and GBS were limited. However in recent years anti-ganglioside antibody tests are available and help to identify large group of neuropathies. Some studies have shown the occurrence of $C$. jejuni enteritis in $13 \%-72 \%$ of patients with GBS [28] [29]. In one study C. jejuni was reported to be present in 18 (26\%) of 43 cases of GBS patients [30]. In a recent study a statistically significant association of both C. jejuni and GBS was reported [31]. The association of $C$. jejuni with GBS seems to vary in different geographic regions. In northern China, the association was seen in $66 \%$ of cases whereas in Europe only $15 \%$ was reported [32]. Serological or culture evidence of a recent $C$. jejuni infection ranged from $23 \%-45 \%$ in a series of patients with GBS in the United Kingdom, Netherlands, United States and Japan [33]-[35]. C. jejuni infection appears to be closely associated with the axonal forms of GBS as reported from studies carried out in China and Japan. Li et al. [36] described $C$. jejuni induced neuritis in chickens mimicking acute axonal neuropathy as seen in human AMAN (acute motor axonal neuropathy) when fed with $C$. jejuni O:19. But this association is not consistent in western countries where the cases of AMAN with antecedent $C$. jejuni infection are less than $10 \%$. The reason for this discrepancy is unknown [35].

Both IgG and IgM antibodies against various ganglosides were detected in the present study. GM1, GD1a and GT1b known to be associated with GBS were found in our patients with GBS. But even in patients with non-paralytic neurological ailments different kinds of anti-ganglioside antibodies were present. The main triggering agents for anti-ganglioside antibodies are $C$. jejuni and celiac diseases. In celiac disease, antibodies to ganglioside are elevated because gliadin can cross-link to ganglioside in a transglutaminase independent manner probably because gliadin specific T-cell present these antigens to the immune system. The limitation of this study is that celiac disease was not ruled out in the patients in the present study. This could have been one of the factors for the presence of anti-ganglioside antibodies, particularly in NC patients. Further research involving patients with celiac disease would be very much useful to understand this aspect. From the study it is concluded that high levels of anti-gangliosides antibodies were present in the GBS patients and their non-paralytic neurological control patients which could be triggered by $C$. jejuni and other factors.

\section{Acknowledgements}

The authors are grateful to Mr. Babbar Raja for technical help, Dr. Ajay Prakash for statistical evaluation and Mr. Gurinder Singh Cheema for helping with the manuscript related work.

\section{Funding}

This work was supported by a grant from the Indian Council of Medical Research, New Delhi, India.

\section{Disclosures}

No conflict of interest

\section{References}

[1] Hughes, R.A.C., Cornblath, D.R. (2005) Guillain-Barré Syndrome. Lancet, 366, 1653-1666. http://dx.doi.org/10.1016/S0140-6736(05)67665-9

[2] Kinnunen, E., Junttila, O., Haukka, J. and Hovi, T. (1998) Nationwide Oral Poliovirus Vaccination Campaign and the Incidence of Guillain-Barré Syndrome. American Journal of Epidemiology, 147, 69-73. http://dx.doi.org/10.1093/oxfordjournals.aje.a009369 
[3] Rees, J. and Hughes, R. (1994) Guillain-Barré Syndrome after Measles, Mumps and Rubella Vaccine (Letter; Comment). Lancet, 343, 733. http://dx.doi.org/10.1016/S0140-6736(94)91614-4

[4] Newton, N. and Janati, A. (1987) Guillain-Barré Syndrome after Vaccination with Purified Tetanus Toxoid. Southern Medical Journal, 80, 1053-1054. http://dx.doi.org/10.1097/00007611-198708000-00028

[5] Winer, J.B., Hughes, R.A.C., Anderson, M.J., Jones, D.M., Kangro, H. and Watkins, R.P.F. (1998) A Prospective Study of Acute Idiopathic Neuropathy. II. Antecedent Events. Journal of Neurology, Neurosurgery \& Psychiatry, 51, 613-618. http://dx.doi.org/10.1136/jnnp.51.5.613

[6] Rhodes, K.M. and Tattersfield, A.E. (1982) Guillain-Barré Syndrome Associated with Campylobacter Infection (Letter). British Medical Journal, 285, 652. http://dx.doi.org/10.1136/bmj.285.6336.173

[7] Kuroki, S., Saida, T., Masafumi, N., et al. (1993) Campylobacter jejuni Strains from Patients with Guillain-Barré Syndrome Belong Mostly to Penner Serogroup 19 and Contain $\beta$-N-acetylglucosamine Residues. Annals of Neurology, 33, 243-247. http://dx.doi.org/10.1002/ana.410330304

[8] Kaldor, J. and Speed, B.R. (1984) Guillain-Barré Syndrome and Campylobacter jejuni: A Serological Study. British Medical Journal, 288, 1867-1870. http://dx.doi.org/10.1136/bmj.288.6434.1867

[9] Heidenreich, F. (1998) Autoantibodies Associated with Peripheral Neuropathies. In Conrad, K., et al., Eds., Pathogenic and Diagnostic Relevance of Autoantibodies. Report on the 4th Dresden Symposium on Autoantibodies Held in Dresden on 21-24 October 1998, Pabst Science Publishers, Lengerich.

[10] Pestronk, A. and Choksi, R. (1997) Multifocal Motor Neuropathy: Serum IgM anti-GM1 Ganglioside Antibodies in Most Patients Detected Using Covalent Linkage of GM1 to ELISA Plates. Neurology, 49, 1289-1292. http://dx.doi.org/10.1212/WNL.49.5.1289

[11] Daune, G.D., Farrer, R.G. and Dalakas, M.C. (1992) Sensory Neuropathy Associated with Monoclonal Immunoglobulin M to GD1b Ganglioside. Annals of Neurology, 31, 683-685. http://dx.doi.org/10.1002/ana.410310621

[12] Vaishnavi, C., Behura, C., Prabhakar, S., Sharma, A. and Kharbhanda, P. (2013) Anti-Ganglioside Antibodies in Patients with Guillain-Barré Syndrome and Other Neurological Disorders. Indian Journal of Medical Microbiology, 31, 177-179.

[13] Asbury, A.K. and Cornblath, D.R. (1990) Assessment of Current Diagnostic Criteria for Guillain-Barré Syndrome. Annals of Neurology, 27, S21-S24. http://dx.doi.org/10.1002/ana.410270707

[14] Meyer, W., Schneider, B., Nobile, Orazio, E., Klotz, M., Schlumberger, W. and Stöcker, W. (2002) EUROLINE AntiGanglioside-Profile: A New Membrane Test for Detection of Antibodies against Gangliosides. Autoimmunity Reviews, 1,71 .

[15] Osterman, P.O., Lundemo, G., Pirskanen, R., Fagius, J., Pihlstedt, P. and Siden, A. (1984) Beneficial Effects of Plasma Exchange in Acute Inflammatory Polyradiculoneuropathy. The Lancet, 2, 1296-1298. http://dx.doi.org/10.1016/S0140-6736(84)90819-5

[16] Gorenjac, M. (2004) Clinical and Diagnostic Role of Ganglioside Antibody Testing. Journal of the International Federation of Clinical Chemistry and Laboratory Medicine, 15, Article ID: 150309.

[17] Nobuhiro, Y., Taki, T., Inagaki, F., Kasama, T., Takahashi, M., Saito, K., et al. (1993) A Bacterium Lipopolysaccharide That Elicits Guillain-Barré Syndrome Has a GM1 Ganglioside-Like Structure. The Journal of Experimental Medicine, 178, 1771-1775. http://dx.doi.org/10.1084/jem.178.5.1771

[18] Yuki, N. and Odada, M. (2005) Ganglioside Mimicry as a Cause of Guillain-Barré Syndrome. Current Opinion in Neurology, 18, 557-561. http://dx.doi.org/10.1097/01.wc0.0000174604.42272.2d

[19] Dikmen, P.Y. and Oge, A.E. (2012) Recurrent GBS with GD1a Anti-Ganglioside Antibodies. Journal of the Peripheral Nervous System, 17, 135-137. http://dx.doi.org/10.1111/j.1529-8027.2012.00384.X

[20] Taheraghdam, A., Pourkhanjar, P., Talebi, M., Bonyadi, M., Pashapour, A., Sharifipour, E., et al. (2014) Correlations between Cytomegalovirus, Epstein-Barr Virus, Anti-Ganglioside Antibodies, Electrodiagnostic Findings and Functional Status in Guillain-Barré Syndrome. Iranian Journal of Neurology, 13, 7-12.

[21] Goddard, E.A., Lastovica, A.J. and Argent, A.C. (1997) Campylobacter 0:41 Isolation in Guillain-Barré Syndrome. Archives of Disease in Childhood, 76, 526-528. http://dx.doi.org/10.1136/adc.76.6.526

[22] Takahashi, M., Koga, M., Yokoyama, K. and Yuki, N. (2005) Epidemiology of Campylobacter jejuni Isolated GuillainBarré and Fisher Syndrome in Japan. Journal of Clinical Microbiology, 43, 335-339. http://dx.doi.org/10.1128/JCM.43.1.335-339.2005

[23] Koga, M., Gilbert, M., Takahashi, M., et al. (2006) Comprehensive Analysis of Bacterial Risk Factors for the Development of Guillain-Barré Syndrome after Campylobacter jejuni Enteritis. The Journal of Infectious Diseases, 193, 547-555. http://dx.doi.org/10.1086/499969

[24] Godschalk, P.C.R., Heikema, A.P., Gilbert, M., et al. (2004) The Crucial Role of Campylobacter jejuni Genes in Au- 
toimmune Antibody Induction. Journal of Clinical Investigation, 114, 1659-1665. http://dx.doi.org/10.1172/JCI200415707

[25] Saida, T., Kuroki, S., Hao, Q., Nishimura, M., Nukina, M. and Obayashi, H. (1997) Campylobacter jejuni Isolates from Japanese Species with Guillain-Barré Syndrome. The Journal of Infectious Diseases, 176, S129-S134. http://dx.doi.org/10.1086/513798

[26] Kaur, U., Chopra, J.S., Prabhakar, S., Radhakrishnan, K. and Rana, S. (1986) Guillain-Barré Syndrome: A Clinical Electrophysiological and Biochemical Study. Acta Neurologica Scandinavica, 73, 394-402. http://dx.doi.org/10.1111/j.1600-0404.1986.tb03295.x

[27] Vaishnavi, C. (2001) Campylobacter Enteritis and Extraintestinal Complications. Gastroenterology Today, 4, $189-191$.

[28] Hughes, R.A. and Rees, J.H. (1997) Clinical and Epidemiologic Features of Guillain-Barré Syndrome. The Journal of Infectious Diseases, 176, S92-S98. http://dx.doi.org/10.1086/513793

[29] Hadden, R.D. and Gregson, N.A. (2001) Guillain-Barré Syndrome and Campylobacter jejuni Infection. Journal of Applied Microbiology, 90, S145-S154. http://dx.doi.org/10.1046/j.1365-2672.2001.01363.X

[30] Pradhan, S., Sinha, S., Prasad, N., Jain, D., Pandey, C.M. and Jha, S. (2007) Preceding Infections and Anti-Ganglioside Antibodies in Patients with Guillain-Barré Syndrome: A Single Centre Prospective Case-Control Study. Clinical Microbiology and Infection, 13, 334-337. http://dx.doi.org/10.1111/j.1469-0691.2006.01636.x

[31] Sharma, A., Lal, V., Modi, M., Vaishnavi, C. and Prabhakar, S. (2011) Campylobacter jejuni Infection in GuillainBarré Syndrome; A Prospective Case Control Study in a Tertiary Care Hospital. Neurology India, 59, 717-721. http://dx.doi.org/10.4103/0028-3886.86547

[32] Schmidt-Ott, R., Schmidt, H., Feldmann, S., Brass, F., Krone, B. and Gross, U. (2006) Improved Serological Diagnosis Stresses the Major Role of Campylobacter jejuni in Triggering Guillain-Barré Syndrome. Clinical and Vaccine Immunology, 13, 779-783. http://dx.doi.org/10.1128/CVI.00065-06

[33] Fugimoto, S. and Amako, K. (1990) Guillain-Barré Syndrome and Campylobacter jejuni Infection. The Lancet, 335, 1350. http://dx.doi.org/10.1016/0140-6736(90)91234-2

[34] McCarthy, N. and Giesecke, J. (2001) Incidence of Guillain-Barré Syndrome Following Infection with Campylobacter jejuni. American Journal of Epidemiology, 153, 610-614. http://dx.doi.org/10.1093/aje/153.6.610

[35] Kuwabara, S. (2004) Guillain-Barré Syndrome: Epidemiology, Pathophysiology and Management. Drugs, 64, 597-610. http://dx.doi.org/10.2165/00003495-200464060-00003

[36] Li, C.Y., Xue, P., Tian, W.Q., Liu, R.C. and Yang, C. (1996) Experimental Campylobacter jejuni Infection in the Chicken: An Animal Model of Axonal Guillain-Barré Syndrome. Journal of Neurology, Neurosurgery Psychiatry, 61, 279-284. http://dx.doi.org/10.1136/jnnp.61.3.279

\section{List of Abbreviations}

1. AMAN—Acute Motor Axonal Neuropathy

2. C. jejuni-Campylobacter jejuni

3. GBS-Guillain-Barré Syndrome

4. LOS-Lipo-Oligosaccharide

5. MFS-Miller-Fisher Syndrome

6. MMN—Multifocal Motor Neuropathy

7. NC-Neurological Controls

8. NNC—Non-Neurological Control 
Scientific Research Publishing (SCIRP) is one of the largest Open Access journal publishers. It is currently publishing more than 200 open access, online, peer-reviewed journals covering a wide range of academic disciplines. SCIRP serves the worldwide academic communities and contributes to the progress and application of science with its publication.

Other selected journals from SCIRP are listed as below. Submit your manuscript to us via either submit@scirp.org or Online Submission Portal.
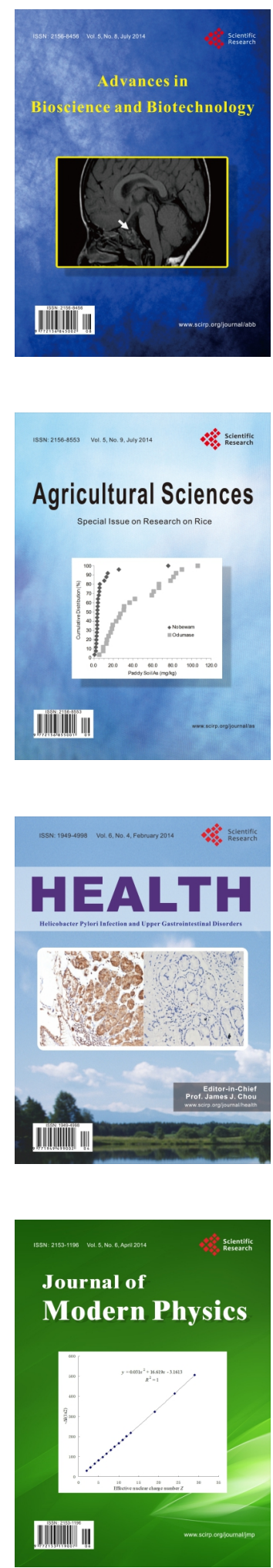
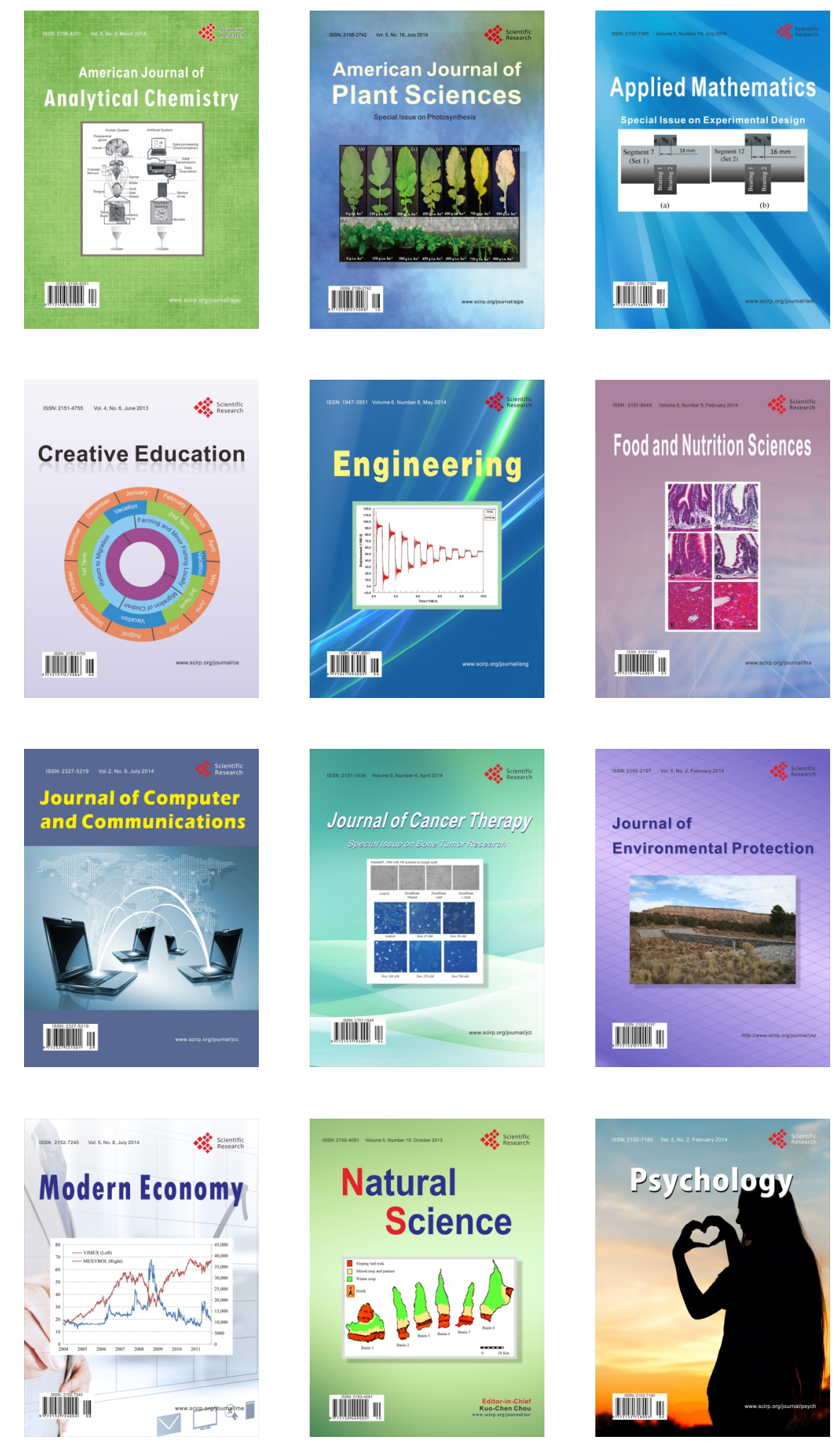